\title{
Transcriptome-wide Identification and Expression Analysis of Brachypodium distachyon Transposons in Response to Viral Infection
}

\author{
Tugba Gurkok*
}

Eldivan Vocational School of Health Services, Department of Medical Services and Techniques, Çankırı Karatekin University, 18100 Cankiri, Turkey

\begin{tabular}{l}
\hline A R T I C L E I N F O \\
Research Article \\
Received 03 April 2017 \\
Accepted 19 July 2017 \\
\hline
\end{tabular}

Keywords:

Brachypodium

Gene expression

Panicum mosaic virus

Transcriptome

Transposon

*Corresponding Author:

E-mail: t.gurkok@gmail.com

\begin{abstract}
A B S T R A C T
Transposable elements (TEs) are the most abundant group of genomic elements in plants that can be found in genic or intergenic regions of their host genomes. Several stimuli such as biotic or abiotic stress have roles in either activating their transcription or transposition. Here the effect of the Panicum mosaic virus (PMV) and its satellite virus (SPMV) infection on the transposon transcription of the Brachypodium distachyon model plant was investigated. To evaluate the transcription activity of TEs, transcriptomic data of mock and virus inoculated plants were compared. Our results indicate that major components of TEs are retroelements in all RNA-seq libraries. The number of transcribed TEs detected in mock inoculated plants is higher than virus inoculated plants. In comparison with mock inoculated plants $13 \%$ of the TEs showed at least two folds alteration upon PMV infection and 21\% upon PMV+SPMV infection. Rather than inoculation with PMV alone inoculation with PMV+SPMV together also increased various TE encoding transcripts expressions. MuDR-N78C_OS encoding transcript was strongly up-regulated against both PMV and PMV+SPMV infection. The synergism generated by PMV and SPMV together enhanced TE transcripts expressions than PMV alone. It was observed that viral infection induced the transcriptional activity of several transposons. The results suggest that increased expressions of TEs might have a role in response to biotic stress in $B$. distachyon. Identification of TEs which are taking part in stress can serve useful information for functional genomics and designing novel breeding strategies in developing stress resistance crops.
\end{abstract}

DOI: https://doi.org/10.24925/turjaf.v5i10.1156-1160.1260

\section{Introduction}

Transposable elements (TEs) are mobile genetic components consisting large portions of eukaryotic genomes (Lisch, 2013; El Baidouri et al., 2014). Since the discovery of transposable elements (TEs) by Barbara McClintock in maize (McClintock, 1950) the origins, roles, and regulation of TEs have been subject to tremendous interest. They have long been assumed as "silent, "junk" or "selfish" DNA; however, recent studies have revealed that most of these repetitive sequences have potential roles in affecting host gene regulation and genomic rearrangements (Lisch, 2013; Ito et al., 2016). In addition several studies have shown that under abiotic or biotic stress TEs are activated or transcribed (Negi et al., 2016). Infections by viruses are one of the main biotic stress types for plants to cope with. Maize Bs1 was the first example of TE which is induced by stress upon barley stripe mosaic virüs (Johns et al., 1985). In addition Grandbastien et al. (1997), reported that, expression of Tnt1 element can be induced after biotic stress treatments in Nicotiana tabacum.
Panicum mosaic virus (PMV) and its satellite virus (SPMV) together infect several crops such as $B$. distachyon (Mandadi and Scholthof, 2012). PMV is a single-stranded, positive-sense RNA virus and host limited to the Poaceae family. Since SPMV is a satellite virus, its replication and movement depends on its helper virus PMV (Scholthof, 1999). As a member of Poaceae $B$. distachyon which has a small size of $272 \mathrm{Mb}$ was introduced as a model plant for genomic and transcriptomic studies. International Brachypodium Initiative (2010) indicated that retrotransposon sequences comprise $21.4 \%$ of the Brachypodium genome, whereas DNA transposons comprise $4.77 \%$ of the genome and it was also defined that while RNA TEs sequences concerted at the centromeres, DNA TEs sequences are located in genic regions. Recently, Mandadi and Scholthof (2012) infected Brachypodium with PMV and SPMV and they reported that PMV+SPMV infection induced more severe disease symptoms than PMV alone and also indicated that it's important for studying host 
pathogen interactions and synergism. Nevertheless the molecular response underlying these symptoms still needs more research.

The dynamics between TEs and their plant hosts depends on a continuous battle and it's hard to declare a winner. But it can be said that TEs often overcome host defenses, at least on evolutionary timescales, because most plant genomes consist large portions of TEs (Bousios and Gaut, 2016). It was previously indicated that the orientation between TE and their hosts conducted with several epigenetic mechanisms (Matsunaga et al., 2012). In this present study, to understand the differences in TE expression following infection with PMV and PMV+SPMV three RNA-seq libraries were analyzed and in silico identification of TEs were accomplished. The results showed that after virus infection specific TE expressions were highly increased in comparison with mock plants. Differential expression patterns between mock and inoculated plants serves important clues about the roles of TEs in virus infection in the model plant Brachypodium.

\section{Material and Methods}

\section{Data Retrieval}

To find out the TEs in mock, PMV and PMV+SPMV inoculated plants three transcriptome libraries assessed for B. distachyon were used. The Illumina RNA-HiSeq reads sequences were obtained in NCBI Short Read Archive. The mock, PMV and PMV+SPMV reads sequences were in retrieved from NCBI Short Read Achieve (SRA) database (https://www.ncbi.nlm.nih.gov/sra) under accession number SRX746906, SRX747740 and SRX747746, respectively (Mandadi and Scholthof, 2015). The reads aligned to the $B$. distachyon whole genome v3.1 (https://phytozome) with Bowtie (Langmead et al., 2009).

\section{Transcriptome-wide TE Identification}

To identify the transposable elements in three assembled sequences we initially ran a homology-based repeat prediction using RepeatMasker (Jurka et al., 2005) with default parameters against the TE database Repbase (version 20160829). Using Plant Genome and Systems Biology (PGSB) database the classification of TEs was performed. The comparison of read numbers between three libraries was evaluated.

\section{Detection of Differentially Expressed TEs}

Following mapping the reads to the genome, for detecting the differentially expressed transposons transcripts .sam files were used in EpiCenter software program (Huang et al., 2011) with default parameters. Reciprocal comparisons were conducted among RNA-seq libraries. TEs representing at least two fold alterations between two libraries were considered as differentially expressed. Then basic local alignment search tool (BLAST) analysis was conducted to annotate the differentially expressed TEs in B. distachyon.

\section{Results}

In this study in silico identification of TEs and differentially expressed TEs in transcriptome libraries of mock and virus infected $B$. distachyon plants were performed. Transcriptome analysis of mock, PMV and PMV+SPMV inoculated B. distachyon plants revealed that the read numbers of TEs were 718.145, 585.723 and 617.644 , respectively (Figure 1). When read numbers of TEs were taken into account it can be said that TEs expressed higher in mock inoculated plants than virus infected plants. The read numbers for TE subfamilies yielded different distributions between DNA and RNA transposons. A slight reduction was observed in the read numbers of RNA TEs after inoculation with virus. Interestingly, after inoculation with PMV and PMV+SPMV while En-Spm and TcMaR DNA TEs showed incremental ratios, LTR, Copia and Gypsy TEs revealed a declined pattern in addition, there was a regular decrement in the read numbers of RNA transposons (Figure 1). Among DNA transposons, hAT, Stowaway, Tourist and Helitron families were not transcriptionally active. Also in retrotransposons LINE and SINE families showed no significant transcription activity.

To elucidate the transcriptional activity of TEs in virus infection, differentially expressed transposons were also determined in $B$. distachyon RNA-seq libraries. An analysis of RNA-seq reads using Epicenter program yielded different expression profiles. As a result of this analyze, at least two fold change alterations taken into account and it was found that 2325 transcripts expressions were up-regulated in both PMV and PMV+SPMV inoculated plants when compared to mock inoculated plants (Figure 2). The increase in TE expression was not only found between mock and inoculated plants but also between PMV and PMV+SPMV inoculated plants (Figure 3, Table 2). The comparison between PMV and mock inoculated plants $13 \%$ (1038) of the TEs were showed $> \pm 2$ fold change. While 943 transcripts revealed up-regulation only $95 \mathrm{TE}$ transcripts revealed downregulation. The fold change ranged between +10 and -4 folds (Table 2). Among differentially expressed TE transcripts 71 of them were annotated and $78 \%$ of these were found to be DNA transposons. The most strongly up-regulated DNA transposons were $M u D R-N 78 C \_O S(8$ fold), DNA-TA-1_BDi and EnSpm-2_BDi (seven folds) (Table 1) on the other hand the most strongly downregulated DNA TE family was $M u D R-N 92 \_O S$ (four folds). Furthermore all the RNA TE expressions were induced after inoculation with PMV. The most induced RNA transposons were Copia-10_BD-I and Copia$36 \_B D-I$ with seven folds.

The results of SPMV+PMV vs Mock comparison showed that $21 \%$ (1287) of transcripts were differentially regulated. The number of transcripts showing up and down-regulation was 1127 and 160, respectively. The highest fold change among up-regulated transcripts was found to be 11 and the lowest was 4 . Of these 91 of differentially regulated TE transcripts were annotated and $79 \%$ of them detected as DNA transposons. Besides, among annotated transcripts $93 \%$ were detected as upregulated. Amongst differentially expressed DNA TE 
transcripts $M u D R-N 78 C_{-} O S$ transposon was up-regulated 10 folds and Mariner-1_BDi was up-regulated 7 folds (Table 1). In addition RNA TEs such as Copia-36_BD-I, COPIA2-I_OS and Gypsy-40_BD-LTR retrotransposons were the most up-regulated with seven folds.
Also to investigate the synergism effect the comparison between SPMV+PMV and PMV alone was carried out. 22 differentially expressed transcripts were assigned. All the transcripts exhibited increased expression profiles; however, only one transcripts annotation could be done.

\section{Read numbers of TEs}

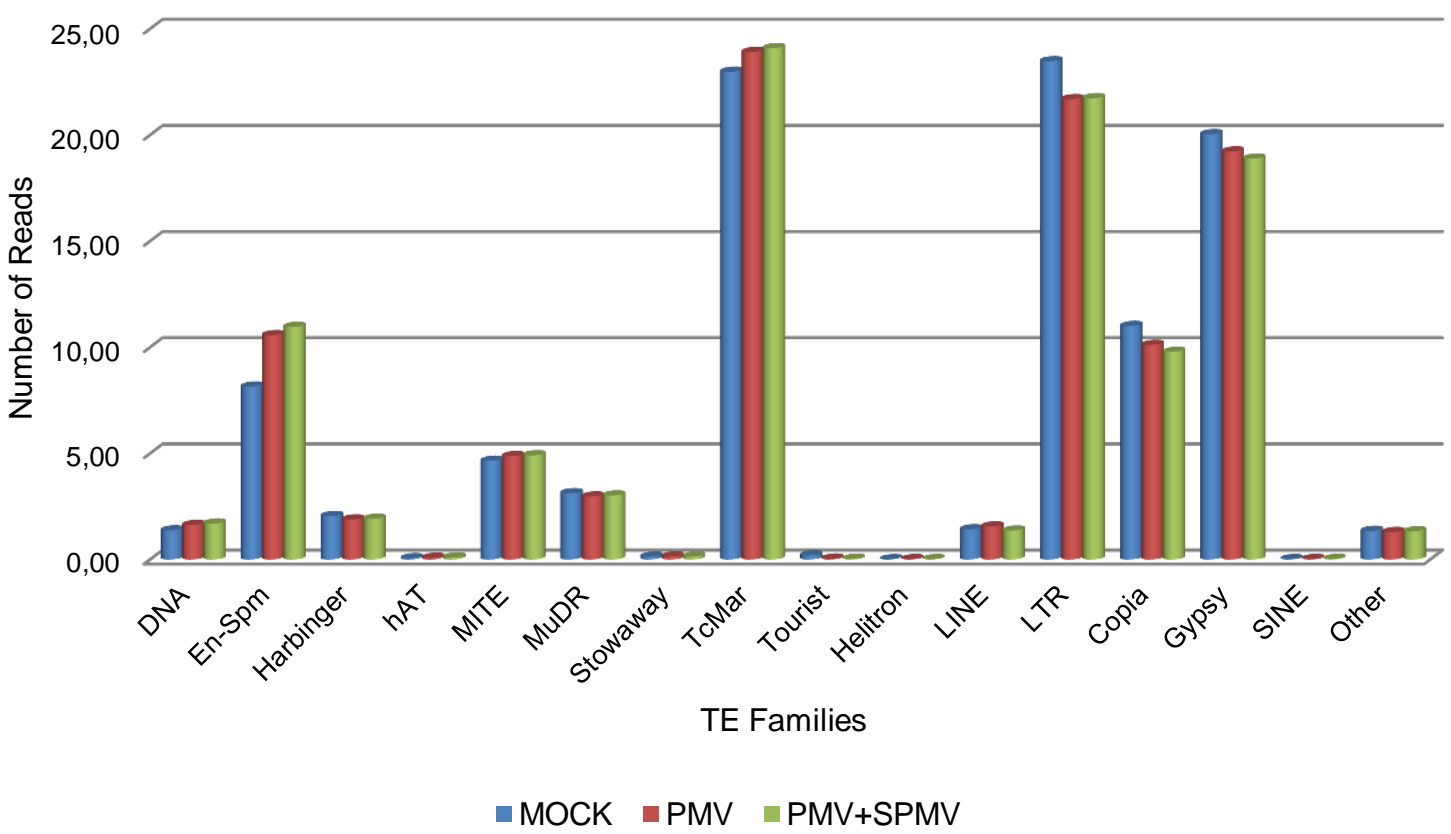

Figure 1 The ratio of read numbers of DNA and RNA transposons obtained from RNA-seq libraries of mock, PMV and PMV+SPMV inoculated plants

Table 1 The most up-regulated TEs found in the $B$. distachyon against viral infection

\begin{tabular}{l|ccc}
\hline \multicolumn{1}{c|}{ Library } & Query id & Transposable element & Fold change \\
\hline PMV+SPMV vs MOCK & Bd1_13466131_2079 & MuDR-N78C_OS & 10 \\
PMV vs MOCK & Bd2_53102462_2380 & MuDR-N78C_OS & 8 \\
PMV vs MOCK & Bd4_47072381_1759 & Copia-10_BD-I & 7 \\
PMV vs MOCK & Bd1_44063680_1718 & Copia-36_BD-I & 7 \\
PMV vs MOCK & Bd2_41397416_1322 & DNA-TA-1_BDi & 7 \\
PMV vs MOCK & Bd1_9508868_843 & EnSpm-2_BDi & 7 \\
PMV+SPMV vs MOCK & Bd4_37643317_11970 & Copia-36_BD-I & 7 \\
PMV+SPMV vs MOCK & Bd1_17433049_1149 & Mariner-1_BDi & 7 \\
PMV+SPMV vs MOCK & Bd2_14466887_1292 & Mariner-1_BDi & 7 \\
PMV+SPMV vs MOCK & Bd1_25513498_4131 & COPIA2-I_OS & 6 \\
PMV+SPMV vs PMV & Bd5_1768716_900 & Mariner-1_BDi & 2 \\
\hline
\end{tabular}

Table 2 The number of differentially expressed transcripts encoding transposable elements

\begin{tabular}{l|ccc}
\hline \multicolumn{1}{c|}{ Library } & Fold change range & Number of upregulated TEs & Number of downregulated TEs \\
\hline \multirow{3}{*}{ PMV vs MOCK } & $2-4$ & 806 & 95 \\
& $5-7$ & 116 & - \\
\hline \multirow{2}{*}{ PMV+SPMV vs MOCK } & $8-10$ & 21 & - \\
& $2-4$ & 986 & 160 \\
\hline PMV+SPMV vs PMV & $5-7$ & 119 & - \\
\hline
\end{tabular}




\section{Discussion}

There is a garnering support that genome and transcriptome orientation via transposon activity might have a role in adaptation and regulation of host stress response (Negi et al., 2016). Besides, regulation of epigenetic mechanisms has an impact on transcriptional activity of TEs in the pathogen infection (Yu et al., 2013; Zhu et al., 2016). The current study revealed a strong induction in TE expression in $B$. distachyon. The read numbers of TEs detected in this study were almost sufficient since the libraries used have high transcriptome reads such as $1.2 \mathrm{~Gb}$ (Mandadi and Scholthof, 2015) so it was able to find most of the transposons in the libraries. More than $25 \%$ of the B. distachyon reference genome is composed of TEs (International Brachypodium Initiative, 2010) and our findings were consistent with the reference genome. International Brachypodium Initiative (2010) reported that retrotransposons found more abundant than DNA transposons in B. distachyon genome; in this study the ratio of transcripts encoding retrotransposons was higher than DNA transposons in all analyzed libraries (Figure 1).

Transcriptional activity of quiescent retrotransposons reported to be activated during wounding and pathogen attack (Grandbastien, 1998). This is consistent with those reports that in PMV and PMV+SPMV inoculated plants; approximately $90 \%$ of the differentially expressed TE transcripts, expressions were increased when compared to mock inoculated plants. There are several studies about stress activated TEs in plants.

Castellano et al. (2016), noted that in cucumber gametophytes, transposable DNA demethylation was observed in virus inoculated plants compared to mock inoculated plants which enhance the transcriptional activity of TEs. It has been reported that methylation modifications in NBS-LRR genes promote or prevent the TE expression (Bernatavichute et al., 2008; Lister et al., 2008). The induction of TEs detected in our study can be explained by the alterations in epigenome against pathogen attack. Besides, it has been reviewed that with the adaptation of retrotransposons to their host genomes they might mimic the promoters of stress induced genes (Todorovska, 2007), hence TEs locations in genome might leads the expression changes.

Nevertheless, although DNA transposon's transcription activity is controlled strongly, several retrotransposons become transcriptionally active under some stresses like pathogen infection, wounding or abiotic stresses (Grandbastien, 1998; Takeda et al., 1998; Capy et al., 2000). But in this current study, it was observed that DNA transposons more active than RNA transposons. In addition retrotransposon classes such as Copia and Gypsy might play an active role in $B$. distachyon metabolism since plenty of transcript encoding them was identified in the libraries (Figure 1).

The analysis revealed that most of the annotated differentially expressed transcripts belong to DNA transposon family. International Brachypodium Initiative (2010) noted that DNA TEs fall into genic regions in $B$. distachyon genome therefore, up-regulation of the DNA transposons in this study can be explained by the presence of the defense genes of these TEs inside or near transcription initiation sites. TE expression regulation can be conducted via various mechanisms. It has been reported that a variety of small RNAs such as microRNAs and short-interfering RNAs (siRNAs) have a function to negatively regulate gene expression and/or to suppress the activity of transposons (Kasschau et al., 2007). The reduction in RNA TEs might be explained by posttranscriptional regulation of transposon transcripts or epigenetic mechanisms like DNA methylation. There might be different regulation mechanisms between DNA and RNA transposons upon different environmental conditions.

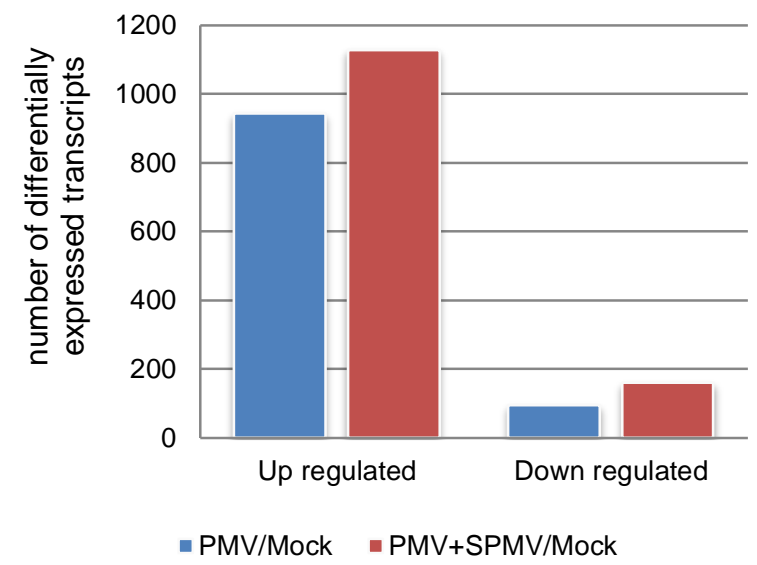

Figure 2 Differentially expressed transcripts between PMV vs Mock and PMV+SPMV vs Mock RNA-seq libraries

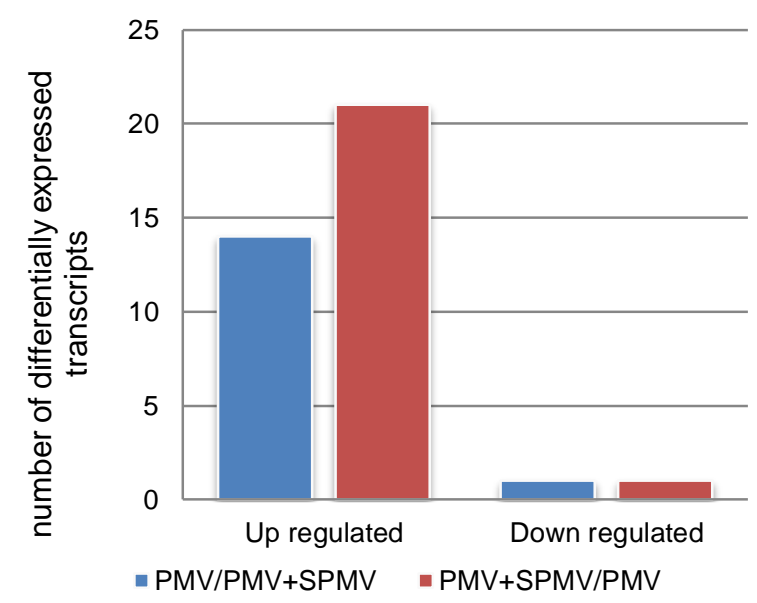

Figure 3 The number of differentially expressed TE transcripts PMV vs PMV+SPMV and SPMV+PMV vs PMV

Here, it was investigated that the number of TE reads was higher in mock inoculated plants than infected plants. DNA methylation, demethylation and histone modifications reported to regulate the TEs expressions in plants (Matzke and Mosher 2014; Duan et al., 2016). Furthermore, DNA methylation mostly arises in TEs and repetitive DNA sequences (Furner and Matzke 2011). Methylation might cause the decreased read numbers of TEs after inoculation with viruses because DNA methylation changes were associated with the stresses and plant immunity (Takeda et al., 1999). Additionally, the 
increase in TE transcript expressions can be explained by methylation changes. MuDR-N78C_OS was the most induced transcript in both comparisons hence $M u D R$ $N 78 C \_O S$ TE might participate as a promoter in stress response. This might highly likely be consequence of epigenetic regulation against virus infection.

\section{Conclusions}

In this present study, pathogen induced transposons were detected and the expressions of them were profiled. As a result of this study it was observed that viral infection induced the transcriptional activity of several transposons. Synergism of PMV and its satellite virus had stronger effect on TE expression than PMV alone. Following the annotation of TEs in B. distachyon against virus infection, it was realized that the annotation has not been fully determined yet and new annotations are requisite for $B$. distachyon. Identification of TEs which are taking part in stress can serve useful information for functional genomics and designing novel breeding strategies in developing stress resistance crops.

\section{References}

Bernatavichute YV, Zhang X, Cokus S, Pellegrini M, Jacobsen SE. 2008. Genome-wide association of histone H3 lysine nine methylation with CHG DNA methylation in Arabidopsis thaliana. PloS One 3(9): e3156.

Bousios A, Gaut BS. 2016. Mechanistic and evolutionary questions about epigenetic conflicts between transposable elements and their plant hosts. Current Opinion in Plant Biology 30: 123-133.

Capy P, Gasperi G, Biémont C, Bazin C. 2000. Stress and transposable elements: co-evolution or useful parasites? Heredity 85(2): 101-106.

Castellano M, Martinez G, Marques MC, Moreno-Romero J, Köhler C, Pallas V, Gomez G. 2016. Changes in the DNA methylation pattern of the host male gametophyte of viroid-infected cucumber plants. Journal of Experimental Botany 67(19): 5857 5868.

Duan CG, Wang X, Xie S, Pan L, Miki D, Tang K, Hsu CC, Lei M, Zhong Y, Hou YJ, Wang Z, Zhang Z, Mangrauthia SK, Xu H, Zhang H, Dilkes B, Tao WA, Zhu JK. 2016. A pair of transposon-derived proteins function in a histone acetyltransferase complex for active DNA demethylation. Cell Research 27: 226-240.

El Baidouri M, Carpentier MC, Cooke R, Gao D, Lasserre E, Llauro C, Mirouze M, Picault N, Jackson SA, Panaud O. 2014. Widespread and frequent horizontal transfers of transposable elements in plants. Genome Research 24: 831-838.

Furner IJ, Matzke M. 2011. Methylation and demethylation of the Arabidopsis genome. Current Opinion in Plant Biology 14(2): 137-141.

Grandbastien MA, Lucas H, Morel JB, Mhiri C, Vernhettes S, Casacuberta JM. 1997. The expression of the tobacco Tnt1 retrotransposon is linked to plant defense responses. Genetica 100(1-3): 241-252.

Grandbastien, MA. 1998. Activation of plant retrotransposons under stress conditions. Trends in plant science 3(5): 181-187.

Huang W, Umbach DM, Jordan NV, Abell AN, Johnson GL, Li L. 2011. Efficiently identifying genome-wide changes with nextgeneration sequencing data. Nucleic Acids Research 39(19): e130-e130.

International Brachypodium Initiative. 2010.Genome sequencing and analysis of the model grass Brachypodium distachyon. Nature 463: 763-768.
Ito H, Kim JM, Matsunaga W, Saze H, Matsui A, Endo TA, Harukawa Y, Takagi H, Yaegashi H, Masuta Y, Masuda S, Ishida J, Tanaka M, Takahashi S, Morosawa T, Toyoda T, Kakutani T, Kato A, Seki M. 2016. A stress-activated transposon in Arabidopsis induces transgenerational abscisic acid insensitivity. Scientific Reports 6.

Johns MA, Mottinger J, Freeling M. 1985. A low copy number, copia-like transposon in maize. The EMBO Journal 4(5): 1093.

Jurka J, Kapitonov VV, Pavlicek A, Klonowski P, Kohany O, Walichiewicz J. 2005. Repbase Update, a database of eukaryotic repetitive elements. Cytogenetic and Genome Research 110(1-4): 462-467.

Kasschau KD, Fahlgren N, Chapman EJ, Sullivan CM, Cumbie JS, Givan SA, Carrington JC. 2007. Genome-wide profiling and analysis of Arabidopsis siRNAs. PLoS Biology 5(3): e57.

Langmead B, Trapnell C, Pop M, Salzberg SL. 2009. Ultrafast and memory-efficient alignment of short DNA sequences to the human genome. Genome Biology 10(3): R25.

Lisch D. 2013. How important are transposons for plant evolution? Nature Reviews Genetics 14(1): 9-61.

Lister R, O'Malley RC, Tonti-Filippini J, Gregory BD, Berry CC, Millar AH, Ecker JR. 2008. Highly integrated single-base resolution maps of the epigenome in Arabidopsis. Cell 133(3): 523-536.

Mandadi KK, and Scholthof KBG. 2012. Characterization of a viral synergism in the monocot Brachypodium distachyon reveals distinctly altered host molecular processes associated with disease. Plant Physiology 160(3): 1432-1452.

Mandadi KK, and Scholthof KBG. 2015. Genome-Wide Analysis of Alternative Splicing Landscapes Modulated during Plant-Virus Interactions in Brachypodium distachyon. The Plant Cell 27: 71-85.

Matsunaga W, Kobayashi A, Kato A, Ito H. 2012. The effects of heat induction and the siRNA biogenesis pathway on the transgenerational transposition of ONSEN, a copia-like retrotransposon in Arabidopsis thaliana. Plant and Cell Physiology 53(5): 824-833.

Matzke MA, Mosher RA. 2014. RNA-directed DNA methylation: an epigenetic pathway of increasing complexity. Nature Reviews Genetics 15(6): 394-408.

McClintock B. 1950. The origin and behavior of mutable loci in maize. Proceedings of the National Academy of Sciences 36(6): 344-355

Negi P, Rai AN, Suprasanna P. 2016. Moving through the Stressed Genome: Emerging Regulatory Roles for Transposons in Plant Stress Response. Frontiers in Plant Science 7.

Scholthof KBG.1999. A synergism induced by satellite panicum mosaic virus. Molecular Plant-Microbe Interactions 12(2): 163166.

Takeda S, Sugimoto K, Otsuki H, Hirochika H. (1998). Transcriptional activation of the tobacco retrotransposon Tto1 by wounding and methyl jasmonate. Plant Molecular Biology 36(3): 365-376.

Takeda S, Sugimoto K, Otsuki H, Hirochika H. 1999. A 13-bp cisregulatory element in the LTR promoter of the tobacco retrotransposon Tto1 is involved in responsiveness to tissue culture, wounding, methyl jasmonate and fungal elicitors. The Plant Journal 18(4): 383-393.

Todorovska E. 2007. Retrotransposons and their role in plantgenome evolution. Biotechnology \& Biotechnological Equipment 21(3): 294-305.

Yu A, G Lepère, Jay F, Wang J, Bapaume L, Wang Y, Abraham AL, Penterman J, Fischer RL, Voinnet O, Navarro L. 2013. Dynamics and biological relevance of DNA demethylation in Arabidopsis antibacterial defense. Proceedings of the National Academy of Sciences 110(6): 2389-2394.

Zhu QH, Shan WX, Ayliffe MA, Wang MB. 2016. Epigenetic mechanisms: An emerging player in plant-microbe interactions. Molecular Plant-Microbe Interactions 29(3): 187-196. 